\title{
Unconventional Magnetic Properties of Cuprates
}

\author{
J.L. González, E.S. Caixeiro, and E.V.L. de Mello \\ Departamento de Física, Universidade Federal Fluminense, Niterói, RJ 24210-340, Brazil
}

Received on 23 May, 2003.

\begin{abstract}
Recently experiments on high critical temperature superconductors have shown that the doping levels and the superconducting gap are usually not uniform properties but strongly dependent on their positions inside a given sample. We show here that the large diamagnetic signal above the critical temperature $T_{c}$ and the unusual temperature dependence of the upper critical field $H_{c 2}$ with the temperature can be explained taking the inomogeneities and a distribution of different local critical temperatures into account.
\end{abstract}

There are increasing evidences that high critical temperature superconductors (HTSC) are intrinsic inhomogeneous materials. This is probably the cause of several unconventional behavior. In particular, recent magnetic imaging through a scanning superconducting quantum device (SQUID) microscopy has displayed a static Meissner effect at temperatures as large as three times the $T_{c}$ of an underdoped LSCO film[1]. Following up SQUID magnetization measurements on powder oriented YBCO and LSCO single crystals[2, 3] have shown a rather high magnetic response which, due to its large signal and structure, cannot be attributed solely to the Ginzburg-Landau (GL) theory of fluctuating superconducting magnetization $[4,8]$. On the other hand the $H$-T phase diagram of the HTSC possess, in certain cases, positive curvature for $H_{c 2}(T)$, with no evidence of saturation at low temperatures [5]. These lack of saturation at low temperatures may minimize the importance of strong fluctuations of the order parameter.

In this paper we develop a unified view for all these anomalous properties. The basic ideas are[9, 10]: the charge distribution inside a HSCT is highly inhomogeneous and may be divided in two types. A hole-poor branch which represents the AF domains and a hole-rich which characterizes the metallic regions. The width of the metallic distribution decreases with the average doping since usually, the samples becomes more homogeneous as the average doping level or average charge density increases. Due to the spatially varying local charge density, it is also expected that the $T_{c}$, instead of being a single value as in usual metallic superconductors, becomes locally dependent. Therefore a given HTSC compound with an average charge density $n_{m}$ possess a distribution of charge density $n(r)$, zero temperature superconducting gap $\Delta_{0}(r)$ and superconducting critical temperature $T_{c}(r)$ where the symbol $(r)$ means a point inside the sample. In this scenario we identify the largest $T_{c}(r)$ with the the pseudogap temperature $T^{*}$ of the compound[11]. Metallic domains with low (high) doping level have high (low) $T_{c}(r)$. Upon cooling below $T^{*}$ the superconducting regions develop at isolated regions as droplets of rain in the air and, eventually they percolates at the superconducting critical temperature $T_{c}$ of the compound at which superconducting long range order is established.

\section{The Model for the Magnetization}

In order to estimate the $M(B)$ we follow the ideas and the procedures of the Critical State Model (CSM) to each superconducting droplet. Upon applying an external magnetic field, a critical current $\left(J_{c}\right)$ is established which opposes the field as $J_{c}(B)=\alpha(T) / B$ according to Ohmer et al[6].

For simplicity we take these superconducting droplets as cylinders of radius $R$, which is sufficient small in order to have a constant charge density $n$ and consequently the critical temperature $T_{c}(n)$ is the same within such cylinder region (As the temperature decreases, more droplets appear and the superconducting regions increase by aggregation of droplets of different $n$ ). The CSM approach leads to the magnetic field dependence of the magnetization in each small cylinder as[7]:

$$
\begin{aligned}
M_{1}(B)= & -\frac{B}{\mu_{0}} \text { for } B \leq B_{c 1} \\
M_{2}(B)= & -\frac{B}{\mu_{0}}+\frac{4 B^{3}}{5 \mu_{0} B^{* 2}}-\frac{8 B^{5}}{15 \mu_{0} B^{* 4}}, B_{c 1} \leq B \leq B^{*} \\
M_{3}(B)= & -\frac{B}{\mu_{0}}-\frac{4 B^{*}}{15 \mu_{0}}\left(2 \frac{B^{5}}{B^{* 5}}-5 \frac{B^{3}}{B^{* 3}}\right. \\
& \left.-2\left[\frac{B^{2}}{B^{* 2}}-1\right]^{5 / 2}\right) \text { for } B_{*} \leq B \leq B_{c 2}
\end{aligned}
$$


Since $T_{c}(n)$ is constant inside a superconducting cylindrical droplet, the critical fields $\left(B_{c 1}\right.$ and $\left.B_{c 2}\right)$ inside the droplets will have their temperature dependence according to the GL theory, e. g., $B_{c 1}(T)=B_{c 1}(0)\left[\left(1-T / T_{c}(n)\right]\right.$ and $B_{c 2}(T)=B_{c 2}(0)\left[\left(1-T / T_{c}(n)\right]\right.$. Taking the dependence of $T_{c}(n)$ on $n$ as a linear relation, namely $T_{c}(n)=$ $T_{0}-b\left(n-n_{c}\right)$, where $n_{c}=0.05$ is the onset of superconductivity and $T_{0}$ is its maximum value, we arrive at the expressions for the critical fields $B_{c 1}(T, n)$ and $B_{c 2}(T, n)$. A similar functional form is supposed for $B^{*}$ due to the $\alpha(T)$ temperature dependence. Thus,

$$
\begin{aligned}
& B_{c 1}(T, n)=B_{c 1}(0)\left[1-\frac{T}{T_{0}-b\left(n-n_{c}\right)}\right] \\
& B_{c 2}(T, n)=B_{c 2}(0)\left[1-\frac{T}{T_{0}-b\left(n-n_{c}\right)}\right] \\
& B^{*}(T, n)=B^{*}(0)\left[1-\frac{T}{T_{0}-b\left(n-n_{c}\right)}\right] .
\end{aligned}
$$

When a given sample is submitted to an applied external magnetic field $B$, the superconducting droplets with carrier concentration $n$ for which the applied field is higher than their second critical field $B_{c 2}(T, n)=B_{c 2}(0)\left[\left(1-T /\left(T_{0}-\right.\right.\right.$ $\left.b *\left(n-n_{c}\right)\right]$, do not contribute to the sample magnetization. This condition is verified for droplets for which $n>n_{\max }$, where $n_{\max }\left(B_{c 2}\right)=n_{c}+T_{0} / b-(T / b) /\left[1-B / B_{c 2}(0)\right]$ is obtained inverting Eq.5. Since $T_{c}(n)$ is a decreasing function of $n$, only the droplets with $n$ bigger than $n_{\max }$ do not contribute to the sample's magnetization because their superconductivity is destroyed by the field $B$. Thus we expect that

$$
M(T, B)=\int_{n_{c}}^{n_{\max }\left(B_{c 2}\right)} P(n) M(B, T, n) d n .
$$

Where $P(n)$ is the distribution function for the charge level inside a given HTCS inferred in Ref.[10]. However, in the context of the CSM, depending on the intensity of the applied field, there are different possibilities in which each domain contributes to $M(B)$. In the low field regime the superconducting clusters will contribute to the magnetization of the sample in three forms: there are some clusters, which are not penetrated by the field $B$, that is $B \leq B_{c 1}(T, n)$ and they contribute to the magnetization with perfect diamagnetism (Eq.1). These clusters have their carrier concentration in the interval, $n<n c+T_{0} / b-(T / b) /\left[1-B / B_{c 1}(0)\right]$. The second group of clusters have their $B_{c 1}(T, n)$ lower than the applied field but $B$ is also lower than $B^{*}(n, T)$. This group is partially penetrated by the field and they contribute to $M(B)$ according Eq.2. These domains have their carrier concentration in the interval $n_{c}+T_{0} / b-(T / b) /[1-$ $\left.B / B_{c 1}(0)\right]<n<n_{c}+T_{0} / b-(T / b) /\left[1-B / B^{*}(0)\right]$. Lastly, there are some superconducting granules for which the applied field is higher than $B^{*}(T, n)$ but also lower than $B_{c 2}(T, n)$. These domains contribute to the magnetization according Eq.3. Therefore, for a sufficient low applied field, the general expression for the $M(B)$ is given by:

$$
\begin{aligned}
M(T, B)= & \int_{n_{m}}^{n_{\max }\left(B_{c 1}\right)} P(n) M_{1}(B, T, n) d n \\
& +\int_{n_{\max }\left(B_{c 1}\right)}^{n_{\max }\left(B^{*}\right)} P(n) M_{2}(B, T, n) d n \\
& +\int_{n_{\max }\left(B^{*}\right)}^{n_{\max }\left(B_{c 2}\right)} P(n) M_{3}(B, T, n) d n(8)
\end{aligned}
$$

The above theory was developed to model the measured magnetization curves of the $\mathrm{La}_{1-x} \mathrm{Sr}_{x} \mathrm{CuO}$ family of compounds. In Fig. 1 we plot the results of our model with the parameter which corresponds to a $n_{m}=0.1$. The qualitative features of the measurements are entirely reproduced and are simply explained by our model; at low fields the perfect diamagnetism is expected for droplets for which the fields are lower than their $B_{c 1}$. We expect $B_{c 1}$ to be weak because the superconducting regions formed above $T_{c}$ are small and isolated and the penetration depth $\lambda$ is large for HTCS. By the same token, the droplets penetrating field $B^{*}$ should not be very strong what decreases rapidly the overall diamagnetic signal for field much weaker than $B_{c 2}$. As the applied field increases, the magnetic response dies off and is reduced to the fluctuations. This is the reason why $M(B)$ has a minimum at very low applied fields. In order to obtain a reliable value of $M(B)$ and to compare with the experimental results, we have incorporated the fluctuation magnetizations induced by the superconducting order parameter, an effect which should be always present, regardless whether the superconductor is more or less inhomogeneous. As noted in reference[7], for superconducting droplets with a homogeneous order parameter and with dimensions $d$ approximately equal to the coherent length $\xi(T)$, the Ginzburg-Landau model provides an exact solution for $M_{\text {fluct }}(B)$. Here we use a simplified "zero-dimensional" for superconducting clusters of radius $d$ smaller or near the coherence length $\xi(T)$, namely[2]:

$$
M_{\text {fluct }}(T, B)=-\frac{2 / 5 k_{B}(\pi \xi T)^{2} B}{\Phi^{2}\left(T / T_{c}-1\right)+(\pi \xi B d)^{2}}
$$

where $k_{B}$ is the Boltzmann constant, $\Phi_{0}$ is the quantum flux and $d \approx \xi \propto\left(T-T_{c}\right) / T_{c}$. This last expression yields a linear $M_{\text {fluct }}(B)$ dependence for low fields and it has been incorporated in our calculations. The specific results for $M_{\text {fluct }}$ are shown in the inset of Fig.1.

We can see that the up-turn field is near $B_{u p}=0.001 \mathrm{~T}$ which agrees with the experimental values[3]. It is worthwhile to mention that previously estimation for the up-turn field considering only the Lawrence-Doniach fluctuations[4] in a layered superconductor[2] yields expected values near $B_{u p}=10 \mathrm{~T}$. These figures bring out the importance of the CSM applied to the superconducting islands above $T_{c}$ to explain the experimental results. 


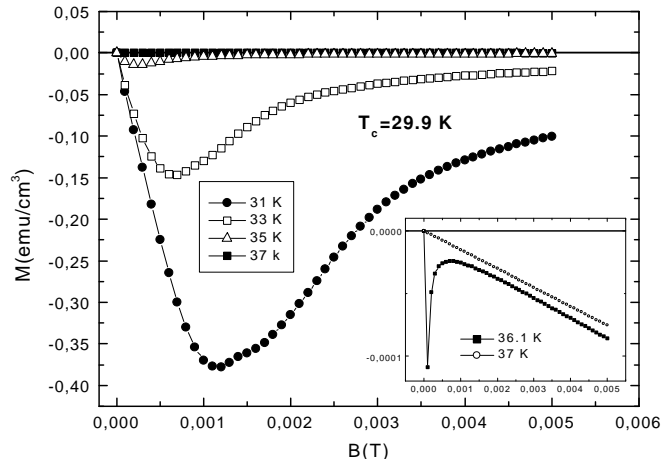

Figure 1. Magnetization for parameters appropriated to the underdoped LSCO as calculated from Eqs. 7-11. The inset shows how the anomolous magnetization is almost vanishing for $T=36.1 \mathrm{~K}$ and evolves into a sole fluctuation regime at $T=37 \mathrm{~K}$. This result is to be compared with the measurements from ref.[3].

\section{The Model for $H_{c 2}$}

In the case of an external magnetic field parallel to the $c$-direction, i.e. perpendicular to the $\mathrm{CuO}_{2}$ planes ( $a b$ direction), the Ginzburg-Landau (GL) upper critical field may be given by[7]

$$
H_{c 2}(T)=\frac{\Phi_{0}}{2 \pi \xi_{a b}^{2}(T)}
$$

where $\Phi_{0}=h c / 2 e$ is the flux quantum and $\xi_{a b}(T)$ is the GL temperature dependent coherence length in the $a b$ plane. In terms of the GL parameters the coher ence length is given

$$
\xi_{a b}^{2}(T)=\frac{\hbar^{2}}{2 m \alpha(T)}=\xi_{a b}^{2}(0)\left(\frac{T_{c}}{T_{c}-T}\right) \quad\left(T<T_{c}\right)
$$

where $\xi_{a b}^{2}(0)=\hbar^{2} / 2 m_{a b} a T_{c}$ is the extrapolated coherence length, $m_{a b}$ is the part of the mass tensor for the $a b$ plane and $a$ is a constant[12]. Therefore,

$$
H_{c 2}(T)=\frac{\Phi_{0}}{2 \pi \xi_{a b}^{2}(0)}\left(\frac{T_{c}-T}{T_{c}}\right) \cdot \quad\left(T<T_{c}\right)(12)
$$

For the LSCO series a coherence length of $\xi_{a b}(0)=30 \AA$ was adopted.

Now, assuming that each isolated or connected superconducting region displays a local $H_{c 2}^{i}$ which is given by the above linearized GL equation, the total $H_{c 2}$ is the sum of these contributions. Since a given local superconducting region " $i$ " has a local temperature $T_{c}(i)$ and probability $P_{i}$, it will contribute to the upper critical field with a local linear upper critical field $H_{c 2}^{i}(T)$ near $T_{c}(i)$. Therefore, the total contribution of the local superconducting regions to the upper critical field is the sum of all the $H_{c 2}^{i}(T)$ 's. Thus, applying Eq.(12), the $H_{c 2}$ for an entire sample is

$$
\begin{aligned}
H_{c 2}(T) & =\frac{\Phi_{0}}{2 \pi \xi_{a b}^{2}(0)} \frac{1}{W} \sum_{i=1}^{N} P_{i}\left(\frac{T_{c}(i)-T}{T_{c}(i)}\right) \\
& =\frac{1}{W} \sum_{i=1}^{N} P_{i} H_{c 2}^{i}(T) \quad\left(T<T_{c}(i) \leq T_{c}(13)\right.
\end{aligned}
$$

where $N$ the number of superconducting regions, or superconducting islands each with its local $T_{c}(i) \leq T_{c}$ and $W=\sum_{i=1}^{N} P_{i}$ is the sum of all the $P_{i}$ 's. As we have already mentioned, at temperatures above $T_{c}$ there are isolated superconducting regions, while below $T_{c}$ these regions percolate and the system may hold a dissipationless current. Since $H_{c 2}$ is experimentally measured at $T<T_{c}(H=0)$, it is the field which destroys the superconducting clusters with $T<T_{c}(i) \leq T_{c}$, leading the system without percolation. The first superconducting regions which are destroyed by the external field are the weakest ones, that is, those which have critical temperatures $T_{c}(i)$ 's lower than the critical temperature $T_{c}(H=0)$.
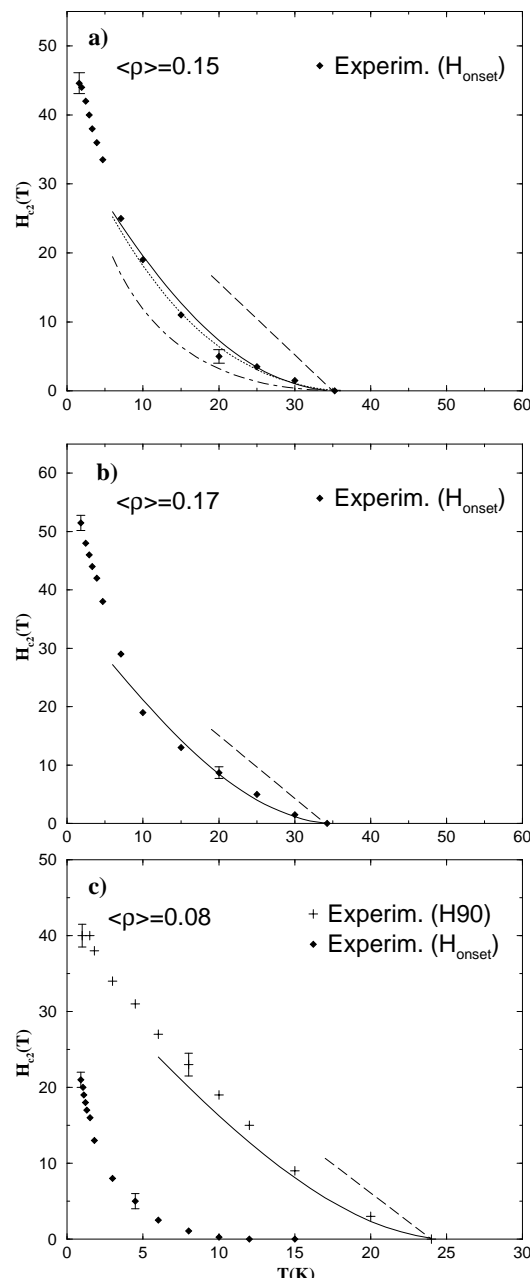

Figure 2. Theoretical results of $H_{c 2}(T)$ (solid lines) of the LSCO series considering the distribution of Ref. [10] together with the experimental data of Ref. [5]. The dashed line is a GL fitting of Eq.(12). In a) the result for a linear normalized distribution (dotted line) is also shown. 
The mechanism is the following: at a temperature $T<$ $T_{c}$ most of the system is superconducting and a small applied field destroys first the superconducting regions at lower $T_{c}(i)$ 's, without loss of long range order. Increasing the applied field causes more regions to become normal and eventually when the regions with $T_{c}(i) \approx T_{c}$ turn to the normal phase, the system is about to have a nonvanishing resistivity. This value of the applied field is taken as the $H_{c 2}$ in our theory, and it is the physical meaning of Eq.(13). Thus, at a given temperature $T$, we sum the superconducting regions with $T<T_{c}(i) \leq T_{c}$, with their respective probabilities.

The experimental upper critical field $H_{c 2}$ of the HTSC may be obtained from the resistivity measurements as it is the field relative to a fraction of the "normal-state" resistivity [5]. By definition, $H_{\text {onset }}$ from the resistive measurements is defined as the magnetic field at which the resistivity $\rho$ first is detected to deviate from the zero in the $\rho$ vs $H$ curves, and this is the assumption used in Eq.(13) and, therefore, it is our definition of $H_{c 2}(T)$. Below we plot $H_{c 2}(T)$ with the measured $H_{\text {onset }}$ for the cases of $n=0.15$ (Fig. 2a) and $n=0.17$ (Fig. 2b) of the LSCO series. For the case of $n=0.08$ (Fig. 2c) we compared our results with $H 90$ since this field vanishes at $T_{c} \approx 24 K$, which is the value of $T_{c}$ obtained from the phase diagram of Ref [10], while $H_{\text {onset }}$ vanishes at $T \approx 12 K$.

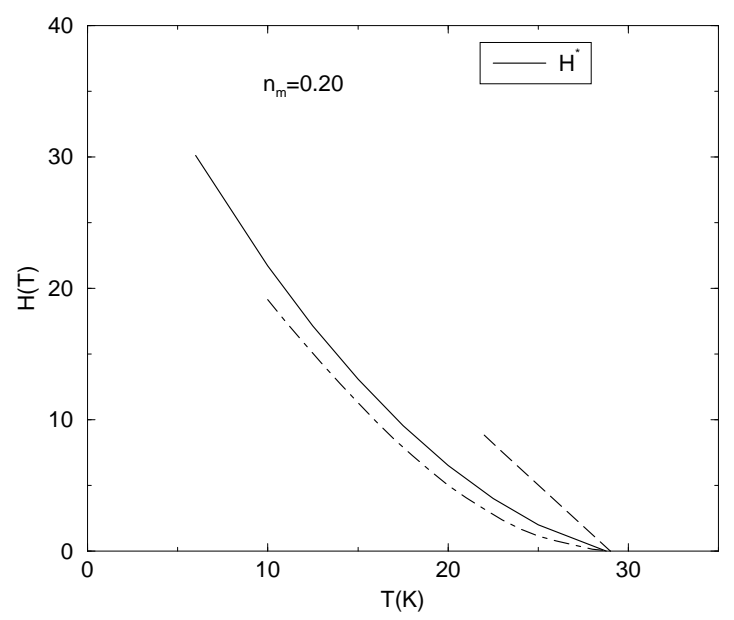

Figure 3. Theoretical results of $H_{c 2}(T)$ (dot-dashed line) for the $n=0.20$ of the LSCO series together with the Nersnt signal measurements curve of Ref. [14] (solid line). The dashed line is a GL fitting.

In Fig. 3 one can see the results for $n=0.20$ compared with $H^{*}$ from the Nernst-signal measurements of Ref. [13, 14]. By the same token, for the Nernst signal[13] measurements of Ref. [14], $H^{*}$ may be considered the upper critical field since it represents an intrinsic field which controls the onset of the flux-flow dissipation and vanishes at a temperature close to $T_{c}$. Therefore, $H^{*}$ may be compared with $H_{\text {onset }}$. Also, in Fig. 2a we plot the results of a linear normalized charge distribution together with the bimodal distribution of Ref. [10]. As one can see, both distributions yield very similar results, which shows that the calculations do not depend on the details of the charge distribution.

\section{Conclusion}

We have been able to reproduce the qualitative features of two nonconventional behavior of HTSC: the unusual diamagnetic signal above $T_{c}$ and the $H_{c 2}$ dependence with the temperature. The basic hypothesis is the non-uniform distribution of charge which was introduced before and which was used to interpret the HTSC phase diagram. Our results demonstrated that the measured normal state magnetization curves, the $B_{c 2}$ fields, Nernst signal and the STM magnetic imaging results may be interpreted through the formation of static superconducting islands at temperatures above the sample's $T_{c}$ and below $T^{*}$.

\section{References}

[1] I. Iguchi, I. Yamaguchi, and A. Sugimoto, Nature, 412, 420 (2001).

[2] A. Lascialfari, A. Rigamonti, L. Romano, P. Tedesco, A. Varlamov, and D. Embriaco, Phys. Rev. B65, 144523 (2002).

[3] A. Lascialfari, A. Rigamonti, L. Romano, A. Varlamov, and I. Zucca, Phys. Rev. Lett. (2003).

[4] C. Baraduc, A. Buzdin, J-Y. Henry, J-P. Brison, and L. Puech, Phys. C248, 138 (1995).

[5] Y. Ando, G.S. Boebinger, A. Passner, L.F. Schneemeyer, T. Kimura, M. Okuya, S. Watauchi, J. Shimoyama, K. Kishio, K. Tamasaku, N. Ichikawa, and S. Uchida, Phys. Rev. B 60, 12475 (1999).

[6] M.C. Ohmer and J.P. Heinrich, J. Appl Phys 44, 1804 (1973).

[7] Michael Tinkham, "Introduction to Superconductivity" McGraw-Hill Inc., New York, 1975.

[8] A. Sewer and H. Beck, Phys. Rev. B64, 014510 (2001).

[9] E.V.L. de Mello, M.T.D. Orlando, E.S. Caixeiro, J.L. González, and E. Baggio-Saitovich, Phys. Rev. B66, 092504 (2002).

[10] E.V.L. de Mello, E.S. Caixeiro, and J.L. González, Phys. Rev. B67, 024502 (2003).

[11] T. Timusk and B. Statt, Rep. Prog. Phys., 62, 61 (1999).

[12] E.S. Caixeiro, J.L. González and E.V.L. de Mello, condmat/0303591, submitted to the Phys. Rev. B.

[13] Z.A. Xu, N.P. Ong, Y. Wang, T. Kakeshita, and S. Ushida, Nature 406, 486(2000).

[14] Y. Wang, N.P. Ong, Z.A. Xu, T. Kakeshita, S. Uchida, D.A. Bonn, and W.N. Hardy, cond-mat/0205299 (unpublished), submitted to Phys. Rev. Lett. 